\title{
Comparative evaluation of sunflower oil and linseed oil as dietary ingredient for gilthead seabream (Sparus aurata) fingerlings
}

\author{
Elham A. Wassef ${ }^{\star}$, Shaymaa H. Shalaby and Norhan E. Saleh \\ Fish Nutrition Laboratory, Aquaculture Department, National Institute of Oceanography and Fisheries (NIOF), Qaiyet-Bey St., Anfoushy, \\ Alexandria, Egypt
}

Received 22 September 2014 - Accepted 9 December 2014

\begin{abstract}
A feeding trial was conducted to define the optimal mixtures of either sunflower oil (SFO) or linseed oil (LO) with fish oil (FO), in fish meal (FM) based diets for gilthead seabream (Sparus aurata) fingerling, without significant effect on fish performance, fatty acid composition and liver structure. The trial lasted nine weeks with 420 fish $(\sim 4.0 \mathrm{~g})$ testing seven isonitrogenous $(\sim 48 \% \mathrm{CP})$ and isolipidic $(\sim 18 \% \mathrm{~L})$ diets contained three incremental inclusions of either SFO or LO $\left(40,48,56 \mathrm{~g} \mathrm{~kg}^{-1}\right)$ and the only-fish oil control (CTRL) diet. Results showed that the combination of $32 \mathrm{~g}$ fish oil plus $48 \mathrm{~g}$ of either SFO or $\mathrm{LO} \mathrm{kg}^{-1}$ diet as the lipid source had performed the best among all. Fatty acid (FA) composition of muscle lipids evidenced that specific fatty acids were selectively retained or utilized. Diet induced- changes in hepatic morphology with vegetable oil inclusion level were further described. Linolenic acid ( $\alpha$-LNA, $n$-3) had led to less pronounced steatosis symptoms than linoleic acid (LOA, $n$-6) in liver cells. This study provides sound support for the use of preferably sunflower oil then linseed oil as a complementary lipid resource $\left(48 \mathrm{~g} \mathrm{~kg} \mathrm{diet}^{-1}\right)$ with marine fish oil in gilthead seabream fingerling diets. Therefore, represents novel data on the potential of using sunflower oil (SFO) as a possible dietary partial substitute of fish oil for the species.
\end{abstract}

Keywords: Sunflower oil / linseed oil / gilthead seabream / Sparus aurata / fatty acids / liver histology

Résumé - Évaluation comparative de l'huile de tournesol et de lin comme ingrédient alimentaire pour les alevins de daurade royale (Sparus aurata). Un essai a été conduit pour définir les mélanges optimaux d'huile de tournesol (SFO, sunflower oil) ou de l'huile de lin (LO, sunflower oil) avec l'huile de poisson (FO, fish oil), dans la farine de poisson (FM, fish meal) des régimes des alevins de daurade royale (Sparus aurata), sans effet significatif sur la performance des poissons, la composition en acides gras et la structure du foie. L'essai a duré 9 semaines et porté sur 420 poissons $(\sim 4.0 \mathrm{~g})$, testant 7 régimes iso-azotés $(48 \% \mathrm{CP})$ et iso-lipidiques $(18 \% \mathrm{~L})$ contenant trois concentrations progressives d'huile de tournesol ou de lin $\left(40,48,56 \mathrm{~kg} \mathrm{~g}^{-1}\right)$ et le contrôle composé uniquement d'huile de poisson (CTRL). Les résultats ont montré que la combinaison de $32 \mathrm{~g}$ l'huile de poisson et de $48 \mathrm{~g}$ d'huile de tournesol ou d'huile de de lin par kilogramme d'aliment représentait la source de lipides la plus performante. La composition en acides gras des lipides musculaires souligne que des acides gras spécifiques ont été sélectivement conservés ou utilisés. Le régime alimentaire a induit des changements de la morphologie hépatique avec des niveaux d'inclusion d'huile végétale décrits ci-après. L'acide linolénique (a-LNA, $n-3$ ) avait conduit à des symptômes de stéatose moins prononcés comparés à l'acide linoléique (LOA, $n-6)$ dans des cellules de foie. Cette étude soutient l'utilisation préférentielle de l'huile de tournesol puis de l'huile de lin comme source de lipides (48 g par kilogramme d'aliment) complémentaire de l'huile de poisson, dans le régime alimentaire de alevins de daurade royale. Ainsi, elle propose de nouvelles données sur le potentiel d'utiliser de l'huile de tournesol (SFO) comme un possible substitut alimentaire des huiles de poissons pour les espèces.

Mots clés : Huile de tournesol / huile de lin / dorade royale / Sparus aurata / acides gras / histologie du foie

\section{Introduction}

The importance of lipids, in aquaculture feeds, as a major source of energy and essential fatty acids neccessary for optimal fish growth is well documented. In marine fish

* Correspondence: elamalywa2007@yahoo.com farming, the fish oil (FO)-added diets, containing high levels of omega-3 longchain polyunsaturated fatty acids ( $\omega$-3 LcPUFA), are considered essential for carnivorous species. However, the demand and price for FO has been increasing with the world wide expanding of aquaculture concomitant with the wild fisheries shortfall in recent years. As sustainable sources of fish oils become more limiting to the expanding demand for 
aquaculture feeds, there is an increasing imperative to identify alternative lipid resources suitable for use in aquaculture feeds. There are a range of alternatives that have already been examined and each has some potential, although few are ideal and most have little if any LcPUFA content (Glencross, 2009). Plant/vegetable oils (VO) are promising and sustainable source as FO-substitutes in aquafeeds, because of their wide availability and competitive price although their use is limited by deficiencies in $\omega-3$ LcPUFA essential for carnivorous species. Extensive research effort on the use of vegetable oils as dietary lipid source in compounded aquafeeds for several marine fish species are already ongoing and are reviewed by Turchini et al. (2009, 2010) and Glencross (2009).

The sparid gilthead seabream, Sparus aurata, is one of the most valuable finfish species commercially produced in Mediterranean mariculture industry where formulated feed is the main production cost. Previous studies on nutrition of this species have highlighted the importance of fish oil as a major constituent in their feeds. However, continuous efforts have been directed towards evaluation of different vegetable oils, either singly or in combination, as FO-substitutes for the species (Alexis, 1997; Benedito-Palos et al., 2008, 2009, 2011; Diaz-Lopéz et al., 2009; El Kerdawy and Salama, 1997; Izquierdo et al., 2003; Menoyo et al., 2004; Wassef et al., 2007, 2009, 2012, 2014). The most commonly investigated vegetable oils, in these earlier research, were: soybean oil (SBO), linseed oil (LO), rapeseed oil (RO), and to a lesser extent palm oil (PO) and olive oil (OO). Linseed oil has an exceptionally favorable fatty acid (FA) composition with a high linolenic acid (LNA, 18:3n-3) content, of nearly $60 \% \alpha$-LNA, compared with $25 \%$ for plant oils generally (Nykter et al., 2006). Both sunflower oil (SFO) and linseed oil (LO) are available worldwide as well as locally, and both are used widely in human food industry, but the latter is more expensive than the former. Despite, LO have already been studied, either singly or mixed, within highly energetic $(25 \% \mathrm{~L})$ fish meal (FM)-based diets (Izquierdo et al., 2003) or plant-protein rich diets (Benedito-Palos et al., 2008) for gilthead seabream, the high level of $n-3$ LNA makes this oil relatively unique in being able provide dietary $n$-3 PUFA at a potentially cost effective rate. On the other hand, reports on using sunflower oil (SFO) as a possible substantial partial substitute for dietary fish oil were mostly limited to Atlantic salmon, Salmo salar (Bell et al., 1993; Bransden et al., 2003; Menoyo et al., 2007; Torestensen et al., 2000), and European seabass, Dicentrarchus labrax (Yildiz and Sener, 2004). Moreover, the high levels of dietary fish oil substitution up to $80 \%$ or $100 \%$ in gilthead seabream feeds had evidenced to result in a remarkably reduced growth performance and feed utilization efficiency and increased feed conversion ratio as well as liver weight and lipid content (Glencross, 2009; Izquierdo et al., 2005; Menoyo et al., 2004; Nasopoulou and Zabetakis, 2012). Nevertheless, besides growth parameters, it is increasingly important to evaluate the effects of FO substitution on fat deposition in liver cells. It is well documented that lipid metabolism is mainly regulated by the liver, including both the synthesis and degradation of fatty acids. Thus, imbalance in the dietary fatty acids (FAs) could modify the functioning and morphology of the organ. In addition, liver functions as a main energy reservoir, frequently in the form of triglycerides (TAG) in gilthead seabream (Kaushik, 1997). Thus, an imbalance in the dietary fatty acids could modify the functioning and morphology of the organ. In fish, high inclusion levels of vegetable oils as a dietary lipid source have also been previously associated to degenerations in liver histological structure (Alexis, 1997), resulting in signs of lipoid liver disease (Caballero et al., 2004). Therefore, the present study was conducted to evaluate the effect of incremental levels of either SFO or LO as dietary FOsubstitutes on growth performance, fish quality (nutrients and muscle FA composition) and liver histological structure of gilthead seabream fingerlings.

\section{Material and methods}

\subsection{Fish and facilities}

A nine-weeks feeding trial was conducted in Fish Nutrition Lab (NIOF), Alexandria, Egypt, with 420 gilthead seabream (S. aurata) fingerlings $\left(\mathrm{BW}_{\mathrm{i}}, 4.00 \pm 0.29 \mathrm{~g} \mathrm{fish}^{-1}\right)$ obtained from a local marine fish hatchery. Fish were acclimatized to laboratory conditions for 2 weeks prior to feeding trial initiation, during which they were fed a commercial diet (40\% CP, $18 \%$ L). Fish were then allocated into 21 tanks $(250 \mathrm{~L}$ each) in groups of 20 fish per tank, as triplicates for seven dietary groups/treatments. Tanks were supplied with constantly flowing natural seawater (10 L min ${ }^{-1}$, salinity $37-39 \mathrm{ppt}$ ) and continuous aeration $\left(\mathrm{DO}>8 \mathrm{mg} \mathrm{L}^{-1}\right)$. During the experimental period mean ambient temperature was $25.3 \pm 0.5^{\circ} \mathrm{C}$ and natural photoperiod was close to $14 \mathrm{~h} \mathrm{day}^{-1}$.

\subsection{Experimental diets}

Seven isonitrogenous $(\sim 48 \% \mathrm{CP})$ and isolipidic $(\sim 18 \% \mathrm{~L})$ fish meal (FM)-based diets were formulated from same ingredients with the exception of the lipid source, and FO was added at a level of $80 \mathrm{~g} \mathrm{~kg}^{-1}$. A control, $100 \%$ FO diet (CTRL) was firstly prepared and then 6 additional diets were prepared by replacement of 50,60 or $70 \%$ of added-FO by either linseed oil (LO) or sunflower oil (SFO) singly in diets designated as LO50 or SFO50, LO60 or SFO60 and LO70 or SFO70 respectively. All diets were produced in the laboratory as extruded pellets as described earlier in details by Wassef et al. (2009), stored at $-20{ }^{\circ} \mathrm{C}$ before being used. Fish were hand fed as two meals per day, six days a week close to apparent satiety, and daily feed intake was recorded. Composition and proximate analysis of experimental diets was given in Table 1. Fatty acid profile of the lipid sources used and of the seven experimental diets were further given in Tables 2 and 3 respectively.

\subsection{Sampling and data collection}

At the beginning and at the end of the experiment, all fish were moderately anaesthetized with cloves oil $\left(0.025 \mathrm{mg} \mathrm{L}^{-1}\right)$ individually measured (Total length, $\mathrm{mm}$ ), weighed (BW, $0.5 \mathrm{~g}$ ) and only at end, liver and viscera were carefully removed and accurately weighed $(0.01 \mathrm{~g})$. Mean initial $\left(\mathrm{BW}_{\mathrm{i}}\right)$ and final body weights $\left(\mathrm{BW}_{\mathrm{f}}\right)$ were then recorded for each 
Table 1. Composition $\left(\mathrm{g} \mathrm{kg}^{-1}\right)$ and proximate analysis $\left(\mathrm{g} \mathrm{kg}^{-1} \mathrm{DM}\right)$ of experimental diets.

\begin{tabular}{|c|c|c|c|c|}
\hline Ingredient & $\begin{array}{c}\text { CTRL } \\
100 \% \mathrm{FO}\end{array}$ & $\begin{array}{l}\text { LO50 / } \\
\text { SFO50 }\end{array}$ & $\begin{array}{l}\text { LO60 / } \\
\text { SFO60 }\end{array}$ & $\begin{array}{l}\text { LO70 / } \\
\text { SFO70 }\end{array}$ \\
\hline Fish meal $(\mathrm{FM})^{1}$ & 540 & 540 & 540 & 540 \\
\hline Soybean meal $(\mathrm{SBM})^{2}$ & 220 & 220 & 220 & 220 \\
\hline Maize flour $^{3}$ & 140 & 140 & 140 & 140 \\
\hline Marine fish oil $(\mathrm{FO})^{4}$ & 80 & 40 & 32 & 24 \\
\hline $\begin{array}{l}\text { Linseed oil (LO) or } \\
\text { sunflower oil }(\mathrm{SFO})^{5}\end{array}$ & - & 40 & 48 & 56 \\
\hline Vit. and Min.mix ${ }^{6}$ & 18 & 18 & 18 & 18 \\
\hline Biogen $^{7}$ & 2 & 2 & 2 & 2 \\
\hline Crude Protein $(\mathrm{CP})$ & 483.0 & 485.4 & 486.7 & 484.3 \\
\hline Total Lipids (TL) & 172.0 & 180.2 & 180.0 & 181.1 \\
\hline Gross energy $\left(\mathrm{MJ} \mathrm{Kg}^{-1}\right)$ & 209.4 & $210.0 / 208.9$ & $209.4 / 212.6$ & 208.5/214.6 \\
\hline
\end{tabular}

\footnotetext{
${ }^{1}$ Danish 999 LT (68.9\% CP, 13.6\% TL). ${ }^{2}$ Local dehulled soybean (Glycine max), Hexane-extracted (44\% CP). ${ }^{3}$ Local Maize, (Zea mays, $9.6 \%$ $\mathrm{CP}, 4.6 \% \mathrm{TL}) .{ }^{4}$ Iceland SR, produced from fresh capelin (Mallotus villosus), herring (Clupea harengus)and/or blue whiting (Micromesistius poutassou). ${ }^{5}$ Local LO or SFO. ${ }^{6} \mathrm{NRC}(1993) .{ }^{7}$ Feed-attractant contains: Alicin $>0.247 \mathrm{M} \mathrm{mole/g}$ and Bacillus ubtilis natto $6 \times 10^{7}$, China Way, Taiwan.
}

dietary group. Hepatosomatic and viscerosomatic indices (HSI and VSI) were calculated, for each individual fish, as the percentage of either liver or viscera mass to fish mass respectively and condition factor $(\mathrm{K})$ as the percentage of total weight to cube of length $\left(100 \mathrm{BW} /\right.$ length $\left.{ }^{3}\right)$.

Fish within each tank were collectively weighed at biweekly intervals to monitor growth rates. No mortality was recorded throughout the experimental period. At the beginning (initial) altogether nine fish per treatment and at the end of the trial three fish from each tank were randomly sampled, and killed by sudden-icing, for biochemical analyses. Muscles was obtained and homogenized under icing (using an EDMUND BÜHLER, HO4/A, Tubingen, Germany) and subjected to proximate composition and fatty acid analyses.

At end of the trial, daily feed consumption was calculated for each treatment. The following growth and feed utilization indices were calculated for all fish in each dietary group $(n=$ 60): total weight gain $(\mathrm{WG}, \mathrm{g} / \mathrm{fish})=\mathrm{BW}_{\mathrm{f}}-\mathrm{BW}_{\mathrm{i}}$, daily weight gain $(\mathrm{DWG}, \mathrm{g} / \mathrm{fish} / \mathrm{d})=\mathrm{WG} / \mathrm{d}$ (days of experiment), feed conversion ratio $[\mathrm{FCR}=($ total feed consumption $($ dry weight, $\mathrm{g}) \times$ (fish biomass increment (live weight, $g)^{-1}$ ], protein efficiency ratio $[\mathrm{PER}=($ fish biomass increment, $\mathrm{g}) \times($ food protein consumption, $\left.\mathrm{g})^{-1}\right]$ and protein productive value $(\mathrm{PPV}=100 \times$ protein gain/protein fed) for the total experimental period. Gross energy (GE) was calculated from the chemical composition using energy conversion factors: $23.4 \mathrm{MJ} \mathrm{Kg}^{-1}$ protein, 39.2 $\mathrm{MJ} \mathrm{Kg}^{-1}$ lipids, and 17.2 $\mathrm{MJ} \mathrm{Kg}^{-1}$ carbohydrates (Goddar, 1996), whereas level of carbohydrates was calculated based on the difference [100 - (water + lipids + protein + ash $)]$ (Shearer, 1994).

\subsection{Analytical procedures}

Proximate analysis of diets and fish muscles were carried out on 3 fish per tank ( 9 per treatment) according to AOAC (1995) standard methodology on wet weight basis. Dry matter content was determined after drying samples into an oven at $105{ }^{\circ} \mathrm{C}$ until constant weight. Crude protein was determined as Nitrogen content $(N \times 6.25)$ by Kjeldahl method, using a semi-automatic Kjeldahl (VELP Scientifica, UDK 126) after acid digestion. Total lipids (TL) content was obtained by extraction of samples with a mixture of chloroform and methanol $(2: 1 \mathrm{v} / \mathrm{v})$ in a Soxhelt apparatus basically as described by Folch et al. (1957). The solvent was removed in a rotary evaporator (BUCHI, Rota vapor R-210/R-215, Flawil, Switzerland), desiccated overnight, and total lipids (TL) was determined gravimetrically. Ash was analyzed by burning oven-dried samples in a muffle furnace at $550{ }^{\circ} \mathrm{C}$ for $16 \mathrm{~h}$.

\subsection{Fatty acids analysis}

Fatty acid (FA) content of tested lipid sources (FO, LO, SFO), and total lipid extracts from diets and fish muscles from each dietary group were determined at start and end of the feeding trial. Fatty acids were converted to methyl esters (FAMS) from at least two aliquots of total lipids each by acid-catalyzed transmethylation with sulphuric acid in methanol overnight at $50{ }^{\circ} \mathrm{C}$ (Christie, 1982). FAMS were purified Thin-Layer Chromatography (TLC) using hexane/diethylether/acetic acid (85:15:15 v/v) as solvent (Tocher and Harvie, 1988). Separation of FAME was conducted in a HP 8690 Gas Liquid Chromatography (GLC) equipped with a flame ionization detector (FID), a cross-linked silica capillary column HP-5 (30 m $\times 0.32 \mathrm{~mm}$, i.d., $0.25 \mu \mathrm{m}$ film thickness) (Hewlett-Packard, CA, USA), and on-column injection and using helium as the carrier gas. Initial temperature programming was as follows: $150{ }^{\circ} \mathrm{C}$, held for $2 \mathrm{~min}$, rising to $200{ }^{\circ} \mathrm{C}$ at $5{ }^{\circ} \mathrm{C} / \mathrm{min}$ and then to $250{ }^{\circ} \mathrm{C}$ and held for $9 \mathrm{~min}$. Peaks were recorded in a personal computer. To identify individual fatty acids an authentic standard FAs (Sigma Chemicals, St. Louis, USA) were used. Results were expressed as weight percentage of each FA to the total fatty acids in the analyzed sample.

The relative abundance of individual fatty acids (FA) of fish oil (FO) and the two tested vegetable oils (linseed oil, LO and sunflower oil, SFO) is given in Table 2. SFO contains higher levels of $n-6$ polyunsaturated fatty acids (PUFA) than 
Table 2. Fatty acid profile (\% total) of the tested lipid sources.

\begin{tabular}{|c|c|c|c|}
\hline $\begin{array}{l}\text { Fatty Acid } \\
\text { (FA) }\end{array}$ & $\begin{array}{l}\text { Fish oil } \\
\text { (FO) }\end{array}$ & $\begin{array}{l}\text { Linseed oil } \\
\text { (LO) }\end{array}$ & $\begin{array}{c}\text { Sunflower oil } \\
\text { (SFO) }\end{array}$ \\
\hline $8: 0$ & 0.1 & $<0.1$ & - \\
\hline $11: 0$ & 0.1 & $<0.1$ & 0.2 \\
\hline $12: 0$ & - & 0.16 & 0.2 \\
\hline 13:0 & 0.1 & 0.2 & 0.1 \\
\hline $14: 0$ & 4.6 & 4.1 & 3.7 \\
\hline $15: 0$ & 0.5 & - & 0.1 \\
\hline $16: 0$ & 6.9 & 3.9 & 10.3 \\
\hline $17: 0$ & 0.2 & - & - \\
\hline 18:0 & 1.9 & 2.9 & 1.3 \\
\hline 20:0 & 0.1 & - & 0.2 \\
\hline 22:0 & $<0.1$ & 0.2 & 0.5 \\
\hline 23:0 & 0.4 & 0.5 & 1.3 \\
\hline $24: 0$ & 0.2 & 0.2 & 0.1 \\
\hline $14: 1$ & 3.3 & 0.4 & 0.2 \\
\hline $15: 1$ & - & - & $<0.1$ \\
\hline $16: 1$ & 9.4 & 0.5 & 0.2 \\
\hline $17: 1$ & 0.4 & - & 0.1 \\
\hline 18:1 & 21.1 & 14.8 & 23.0 \\
\hline $20: 1$ & 8.1 & - & 0.2 \\
\hline $22: 1$ & 9.9 & 0.9 & 0.3 \\
\hline $24: 1$ & 1.1 & 1.1 & 0.9 \\
\hline $18: 2 n-6$ & 1.2 & 10.7 & 56.0 \\
\hline $18: 3 n-3$ & 1.1 & 58.6 & 1.1 \\
\hline $20: 2 n-6$ & 2.0 & - & - \\
\hline $20: 3 n-3$ & 0.8 & - & - \\
\hline $20: 4 n-6$ & 1.4 & - & - \\
\hline $20: 5 n-3$ & 12.7 & - & - \\
\hline $22: 2 n-6$ & 1.1 & - & - \\
\hline $22: 5 n-3$ & 1.1 & - & - \\
\hline $22: 6 n-3$ & 8.3 & - & - \\
\hline SFA & 15.1 & 12.2 & 18.0 \\
\hline MUFA & 53.3 & 17.7 & 24.9 \\
\hline PUFA & 29.7 & 69.3 & 57.1 \\
\hline$n-3$ LcPUFA & 22.9 & - & - \\
\hline n-6 PUFA & 5.7 & 10.7 & 56.0 \\
\hline
\end{tabular}

$n=3, \mathrm{SD}=0.00$ or ranged between $0.001-0.005$; SFA, saturated FA; MUFA, monounsaturated FA; PUFA, polyunsaturated FA; LcPUFA, longchain PUFA.

LO with linoleic acid (LOA, 18:2 n-6) dominating at approximately $56 \%$ of total FAs, while the latter has a higher proportion $(58.6 \%)$ of linolenic acid ( $\alpha$-LNA, 18:3n-3). SFO has a relatively higher content of total saturated (SFA, mainly 16:0) and monounsaturated fatty acids (MUFA, specially 18:1n-9, $n$-7), than LO. Fish oil/CTRL diet contained the highest MUFA and $n-3$ LcPUFA, primarily ecosapentenoic acid (EPA) and docosahexaenoic acid (DHA) and the lowest level of total PUFA, especially the $n-6$ group.

\subsection{Liver histology}

At end of the feeding trial, 3 random liver portions from each fish sampled ( 9 per treatment) were fixed in $10 \%$ neutral formalin solution, dehydrated in ethanol series, embedded in paraffin wax, cross-sectioned at $4 \mu \mathrm{m}$, stained with Hematoxylin and Eosin ( $\mathrm{H}$ and $\mathrm{E}$ ), and light-microscopically examined at magnification $\times 400$ and $\times 1000$.

\subsection{Statistical analysis}

All results are presented as means standard deviation (SD) ( $n=60$ for growth performance-, $n=9$ for proximate composition- and $n=3$ for fatty acid-analyses). Statistical testing to verify differences between control group and each of the 3 inclusion level of either vegetable oil separately (i.e. CTRL, LO50, LO60, LO70 or, CTRL, SFO50, SFO60, SFO70) was carried out using a one-way ANOVA followed by Tukey's multiple comparison test. Differences between means was reported significant if $P \leq 0.05$ and highly significant if $P \leq 0.01$, and the former level was only considered for fatty acid composition of fish muscles. The statistical analysis was performed using the SPSS package, version 16 (SPSS Inc., Chicago, IL, USA).

\section{Results}

\subsection{Fatty acid composition of tested lipid sources and experimental diets}

The content of total lipids (TL) in all experimental diets was almost similar (17.2-18.1\% DM), but their fatty acid composition were clearly different (Tab. 3). Linoleic acid (LOA), linolenic acid ( $\alpha$-LNA), ecosapentenoic acid (EPA) and docosahexaenoic acid (DHA) were strongly influenced by diet treatment. As expected, total $n-3$ LcPUFA content gradually decreased and $n-6$ PUFA gradually increased within the experimental diets with each elevation of either LO or SFO incorporation level. Estimated amount of $n-3$ LcPUFA in the VO-added diets was $5.5-11.1 \%$ (DM of total FAs), and EPA plus DHA level (3.5-8.4\% respectively) was considered satisfactory to cover the theoretical requirements of this species juvenile. Mainly due to the different LOA and $\alpha$-LNA contents of the plant oil used, the $n-3 / n-6$ ratio markedly differed among the experimental diets, ranging from 0.27 in the SFO70 diet to 3.78 in LO50 diet versus 3.36 of CTRL diet (Tab. 3).

\subsection{Growth performance and feed utilization efficiency}

Growth and feed utilization and indices of gilthead seabream fed the experimental diets over 9 weeks are shown in Table 4. It is obvious that, all diets were well consumed by experimental fish regardless of their lipid source or inclusion level. Dietary LO or SFO incorporation had no significant $(P>0.05)$ effect on total or daily feed intake, (FI or DFI respectively), however, both values were the lowest in fish fed the highest level of VO. As a general observation, SFO-fed fish performed better growth and feed utilization, at all inclusion levels, than the corresponding of LO-fed fish, indicating a relatively better efficacy and utilization of the former diets than the latter in this context. The highest final body weight $\left(\mathrm{BW}_{\mathrm{f}}\right)$, live weight gain $(\mathrm{WG})$ and the lowest feed conversion ratio (FCR) were recorded for fish fed the SFO50 then LO50 diets $\left(40 \mathrm{~g} \mathrm{~kg}^{-1}\right.$ diet $)$ comparable $(P>0.05)$ to those of CTRL fish. Despite the significant $(P<0.05)$ reduction in weight gain (WG) observed when fish fed either SFO60 or LO60 diet 
Table 3. Fatty acid (FA) profile (\% total) of the experimental diets.

\begin{tabular}{|c|c|c|c|c|c|c|c|}
\hline FA & CTRL & LO50 & LO60 & LO70 & SFO50 & SFO60 & SFO70 \\
\hline $14: 0$ & 3.8 & 1.7 & 1.2 & 1.0 & 1.5 & 1.5 & 1.2 \\
\hline $15: 0$ & 0.5 & 0.3 & 0.3 & 0.2 & 0.5 & 0.5 & 0.7 \\
\hline $16: 0$ & 10.7 & 8.9 & 7.6 & 6.8 & 12.3 & 11.6 & 10.1 \\
\hline $17: 0$ & 0.3 & 0.3 & 0.2 & 0.1 & 0.3 & 0.2 & 0.1 \\
\hline 18:0 & 5.1 & 5.7 & 5.4 & 3.7 & 3.2 & 2.8 & 2.1 \\
\hline 20:0 & 0.3 & 0.1 & 0.1 & - & 0.3 & 0.3 & - \\
\hline 22:0 & 0.4 & 0.5 & 1.8 & 0.1 & 0.4 & 0.6 & 0.4 \\
\hline 23:0 & $<0.1$ & - & - & 1.8 & - & - & 1.2 \\
\hline 24:0 & 0.1 & 0.2 & 0.4 & 0.4 & 0.6 & 0.8 & 0.9 \\
\hline 14:1 & 3.1 & 2.1 & 1.6 & 1.2 & 1.1 & 1.0 & 1.0 \\
\hline $15: 1$ & 0.1 & 0.1 & - & - & 0.1 & 0.2 & 0.3 \\
\hline 16:1 & 9.8 & 4.1 & 3.4 & 2.8 & 3.7 & 2.6 & 1.6 \\
\hline $17: 1$ & 0.5 & 0.1 & 0.1 & 0.3 & 0.3 & 0.2 & 0.2 \\
\hline $18: 1 n-9, n-7$ & 21.9 & 16.8 & 16.5 & 14.1 & 23.1 & 23.9 & 26.0 \\
\hline $20: 1$ & 5.9 & 1.7 & 0.6 & 0.7 & 1.9 & 1.0 & 1.2 \\
\hline $22: 1$ & 6.1 & 2.9 & 1.4 & 0.9 & 3.2 & 2.8 & 2.2 \\
\hline $24: 1$ & - & 0.1 & 0.3 & 0.4 & 0.2 & 0.6 & 0.5 \\
\hline $18: 2 n-6$ & 2.2 & 8.7 & 12.7 & 15.1 & 29.3 & 34.6 & 37.3 \\
\hline $18: 3 n-3$ & 1.6 & 29.2 & 34.6 & 39.2 & 2.3 & 3.1 & 4.9 \\
\hline $20: 2 n-6$ & 1.2 & 0.1 & 0.1 & 0.2 & 0.4 & 0.3 & 0.2 \\
\hline $20: 3 n-3$ & 1.3 & 0.7 & 0.6 & 0.4 & 0.4 & 0.3 & 0.2 \\
\hline $20: 4 n-6$ & 1.5 & 0.8 & 0.6 & 0.5 & 0.4 & 0.3 & 0.3 \\
\hline $20: 5 n-3$ & 9.1 & 3.6 & 1.9 & 1.6 & 3.2 & 2.7 & 2.0 \\
\hline $22: 2 n-6$ & 1.4 & 1.0 & 0.8 & 0.8 & 0.3 & 0.2 & 0.1 \\
\hline $22: 5 n-3$ & 1.7 & 2.1 & 1.5 & 1.6 & 2.8 & 2.6 & 1.1 \\
\hline $22: 6 n-3$ & 8.6 & 4.8 & 3.1 & 2.1 & 4.6 & 2.8 & 2.2 \\
\hline SFA & 21.2 & 17.7 & 17.0 & 14.1 & 19.1 & 18.3 & 16.7 \\
\hline MUFA & 47.4 & 27.8 & 23.9 & 20.4 & 33.4 & 32.3 & 33.0 \\
\hline PUFA & 28.6 & 51.0 & 55.9 & 61.3 & 43.7 & 46.9 & 48.3 \\
\hline$n-3$ LcPUFA & 20.7 & 11.2 & 7.1 & 5.7 & 11.0 & 8.4 & 5.5 \\
\hline n-6 PUFA & 6.3 & 10.6 & 14.2 & 16.6 & 30.4 & 35.4 & 37.7 \\
\hline EPA+DHA & 17.7 & 8.4 & 5.0 & 3.7 & 7.8 & 5.5 & 4.2 \\
\hline$n-3 / n-6$ ratio & 3.5 & 3.8 & 2.9 & 2.7 & 0.4 & 0.3 & 0.3 \\
\hline
\end{tabular}

$n=3, \mathrm{SD}=0.00$ or ranged between 0.001-0.005; SFA, saturated fatty acids; MUFA, monounsaturated FA; PUFA, polyunsaturated FA; LcPUFA, longchain PUFA.

(48 $\mathrm{g} \mathrm{VO} \mathrm{kg}^{-1}$ diet), weight gain was almost similar in both VO50 and VO60 dietary groups $(P>0.05)$. This was in agreement with the absence of significant $(P>0.05)$ differences in their daily weight gain (DWG) from CTRL (Tab. 4).

Condition factor $(\mathrm{K})$ and hepatosomatic index (HSI), remained constant $(P>0.05)$, whereas viscerosomatic index (VSI) significantly $(P<0.05)$ increased with the elevation of dietary vegetable oil compared to CTRL fish. Moreover, there were no significant $(P>0.05)$ influence of SFO-diets on feed conversion (FCR) or protein efficiency (PER) ratios, whilst those ratios were both reduced $(P<0.05)$ for only LO70fed fish. Likewise, protein productive value (PPV) was also lowered $(P<0.01)$ in VO60 and VO70 dietary groups, particularly for the LO70-fed fish. Therefore, in terms of growth and feed efficiency, the best performed diets were SFO50 then LO50, with a combination of $40 \mathrm{~g}$ FO plus $40 \mathrm{~g} \mathrm{VO} \mathrm{kg}^{-1}$ diet, with no negative effects at all, followed by SFO60 and LO60, contained $32 \mathrm{~g} \mathrm{FO}$ and $48 \mathrm{~g} \mathrm{VO} \mathrm{kg}^{-1}$ with no significant difference among them (VO50 and VO60). The worst diets among all were those contained only $24 \mathrm{~g} \mathrm{FO}$ and $56 \mathrm{~g} \mathrm{VO} \mathrm{kg}^{-1}$ diet (Tab. 4).

\subsection{Muscle major nutrients composition}

At end of growth trial, protein content (\%CP) of fish muscles showed no major influence $(P>0.05)$ from the diet except for LO60 and LO70-groups, which recorded about $15.6 \%$ and $17 \%$ reduction respectively relative to that of CTRL (Tab. 5). In the mean time, variation in protein content between LO60 and LO50 fish groups was not significant $(P>0.05)$. It is also noted that, protein content of SFO-fed fish was comparatively higher than the corresponding for LO-fed fish, at all inclusion levels, with SFO50 fish having the highest value among all. In contrast, total lipids (TL) increased with each dietary elevation in vegetable oil level, more obvious for LO-fed groups, and differences were significant $(P<0.05)$ for LO70, SFO60 and SFO70 fish relative to CTRL fish. Inclusion of either SFO at levels of 48 and $56 \mathrm{~g} \mathrm{~kg}^{-1}$ diet has resulted in $22.7 \%$ or $28.3 \%$ increase in total lipids after 9 weeks feeding, whereas these percentages were $25.4 \%$ and $44 \%$ for the corresponding LO-fed fish. Muscle ash and moisture contents did not significantly $(P>0.05)$ altered among dietary treatments. The best nutrient values were reported in SFO50 fed fish (176.4 g CP, 

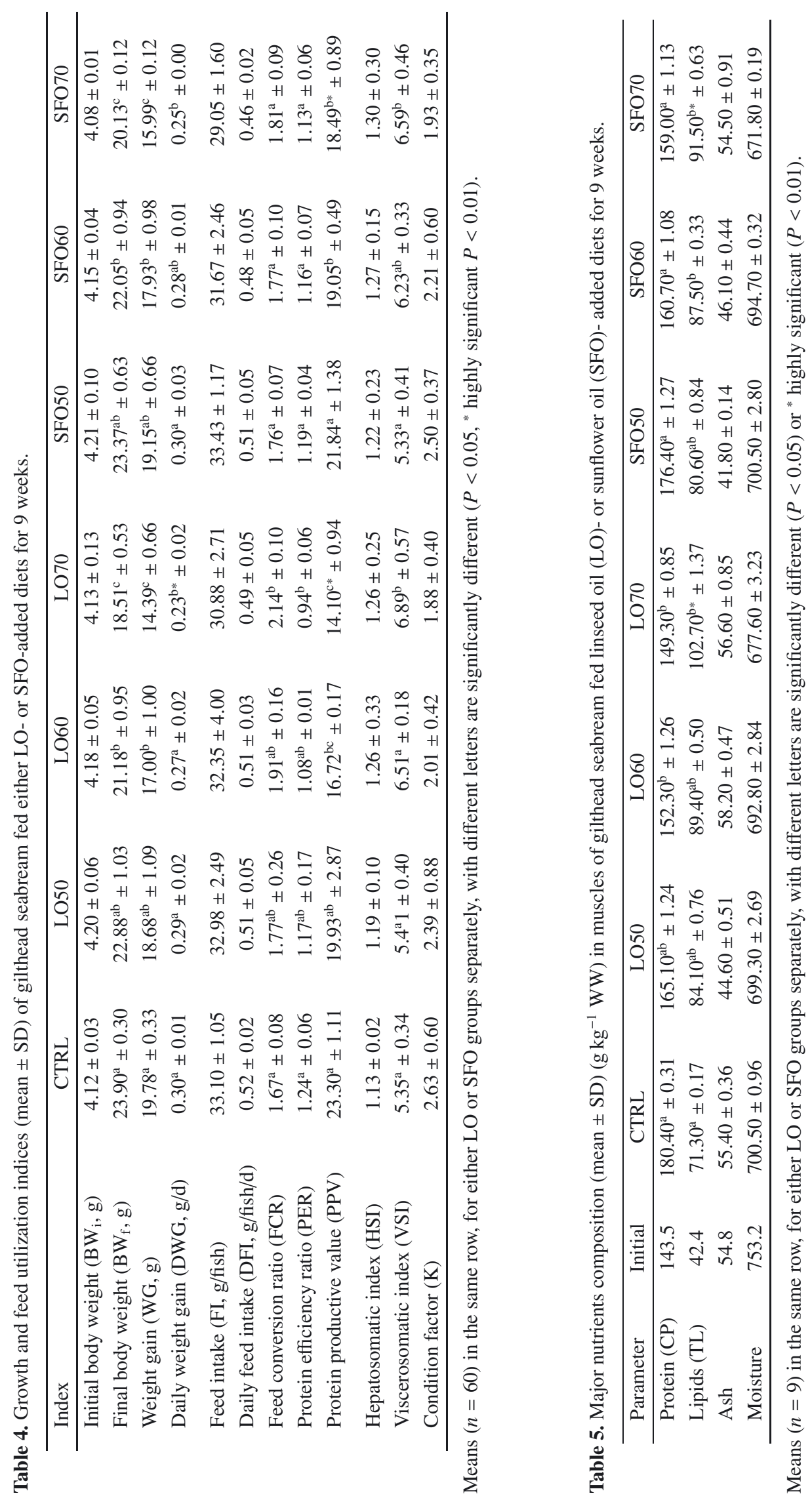
Table 6. Fatty acid (FA) composition (\% total) of muscles of gilthead seabream fed either LO- or SFO-added diets for 9 weeks.

\begin{tabular}{|c|c|c|c|c|c|c|c|}
\hline FA & CTRL & LO50 & LO60 & LO70 & SFO50 & SFO60 & SFO70 \\
\hline $6: 0$ & $0.2^{\mathrm{a}} \pm 0.0$ & - & - & - & $0.1^{\mathrm{b}} \pm 0.0$ & $0.1^{\mathrm{b}} \pm 0.0$ & $0.1^{\mathrm{b}} \pm 0.0$ \\
\hline $8: 0$ & $0.1^{\mathrm{a}} \pm 0.0$ & $0.1^{\mathrm{a}} \pm 0.0$ & $0.1^{\mathrm{a}} \pm 0.0$ & $0.1^{\mathrm{b}} \pm 0.0$ & - & - & - \\
\hline $10: 0$ & $0.2^{\mathrm{a}} \pm 0.0$ & $0.3^{\mathrm{b}} \pm 0.0$ & $0.3^{\mathrm{c}} \pm 0.0$ & $0.2^{\mathrm{ac}} \pm 0.0$ & $0.2^{\mathrm{a}} \pm 0.0$ & $0.1^{\mathrm{b}} \pm 0.0$ & $0.1^{\mathrm{c}} \pm 0.0$ \\
\hline 11:0 & $0.5^{\mathrm{a}} \pm 0.0$ & $0.3^{b} \pm 0.0$ & $0.3^{c} \pm 0.0$ & $0.2^{\mathrm{c}} \pm 0.0$ & $0.1^{\mathrm{b}} \pm 0.0$ & $0.1^{\mathrm{c}} \pm 0.0$ & $0.1^{\mathrm{c}} \pm 0.0$ \\
\hline 12:0 & - & $0.2^{\mathrm{b}} \pm 0.0$ & $0.1^{\mathrm{c}} \pm 0.0$ & $0.2^{c} \pm 0.0$ & $0.1^{\mathrm{a}} \pm 0.0$ & $0.1^{\mathrm{a}} \pm 0.0$ & - \\
\hline 13:0 & - & $0.1^{\mathrm{b}} \pm 0.0$ & $0.1^{\mathrm{b}} \pm 0.0$ & $0.2^{\mathrm{c}} \pm 0.0$ & trace & trace & - \\
\hline 14:0 & $3.6^{\mathrm{a}} \pm 0.5$ & $1.4^{\mathrm{b}} \pm 0.0$ & $2.8^{\mathrm{c}} \pm 0.4$ & $3.1^{\mathrm{ac}} \pm 0.0$ & $1.5^{\mathrm{a}} \pm 0.10$ & $2.0^{\mathrm{b}} \pm 0.1$ & $2.4^{\mathrm{b}} \pm 0.5$ \\
\hline 15:0 & $0.5^{\mathrm{a}} \pm 0.1$ & $0.3^{\mathrm{b}} \pm 0.0$ & $0.3^{\mathrm{b}} \pm 0.0$ & $0.5^{\mathrm{a}} \pm 0.0$ & $0.3^{\mathrm{b}} \pm 0.1$ & $0.2^{\mathrm{b}} \pm 0.0$ & $0.2^{\mathrm{b}} \pm 0.0$ \\
\hline 16:0 & $12.7^{\mathrm{a}} \pm 2.0$ & $16.9^{\mathrm{ab}} \pm 2.0$ & $17.2^{\mathrm{ab}} \pm 2.0$ & $19.4^{b} \pm 1.3$ & $14.7^{\mathrm{ab}} \pm 1.3$ & $16.9^{\mathrm{cb}} \pm 0.6$ & $18.5^{\mathrm{c}} \pm 0.9$ \\
\hline 17:0 & $0.3^{\mathrm{a}} \pm 0.0$ & $0.2^{\mathrm{b}} \pm 0.0$ & $0.3^{\mathrm{a}} \pm 0.0$ & $0.2^{\mathrm{ab}} \pm 0.0$ & $0.2^{\mathrm{b}} \pm 0.0$ & $0.2^{\mathrm{a}} \pm 0.0$ & $0.2^{\mathrm{a}} \pm 0.0$ \\
\hline 18:0 & $6.1^{\mathrm{a}} \pm 1.0$ & $11.2^{\mathrm{b}} \pm 1.0$ & $10.8^{\mathrm{b}} \pm 1.0$ & $10.4^{b} \pm 0.6$ & $7.8^{b} \pm 0.3$ & $8.6^{\mathrm{b}} \pm 0.4$ & $8.8^{\mathrm{b}} \pm 0.2$ \\
\hline 20:0 & $1.2^{\mathrm{a}} \pm 0.0$ & $0.3^{\mathrm{b}} \pm 0.0$ & $0.2^{\mathrm{b}} \pm 0.0$ & $0.2^{\mathrm{cb}} \pm 0.0$ & $0.3^{b} \pm 0.0$ & $0.4^{\mathrm{c}} \pm 0.0$ & $0.2^{\mathrm{d}} \pm 0.0$ \\
\hline 22:0 & $0.3^{\mathrm{a}} \pm 0.0$ & $2.1^{b} \pm 0.0$ & $1.7^{\mathrm{c}} \pm 0.0$ & $0.2^{\mathrm{d}} \pm 0.0$ & $1.7^{\mathrm{b}} \pm 0.0$ & $1.3^{\mathrm{c}} \pm 0.0$ & $1.3^{c} \pm 0.0$ \\
\hline 24:0 & $0.3^{\mathrm{a}} \pm 0.0$ & $0.5^{\mathrm{b}} \pm 0.0$ & $0.4^{\mathrm{ca}} \pm 0.0$ & $0.3^{\mathrm{a}} \pm 0.0$ & $0.8^{b} \pm 0.0$ & $0.4^{\mathrm{c}} \pm 0.0$ & $1.5^{\mathrm{d}} \pm 0.0$ \\
\hline $14: 1$ & $2.2^{\mathrm{a}} \pm 0.5$ & $1.1^{\mathrm{b}} \pm 0.1$ & $1.1^{\mathrm{b}} \pm 0.1$ & $1.0^{\mathrm{b}} \pm 0.0$ & $1.0^{\mathrm{b}} \pm 0.0$ & $1.0^{\mathrm{b}} \pm 0.0$ & $1.0^{\mathrm{b}} \pm 0.0$ \\
\hline $15: 1$ & $0.1^{\mathrm{a}} \pm 0.0$ & $0.10^{\mathrm{a}} \pm 0.0$ & - & $\begin{array}{l}-0.0 \\
-\end{array}$ & $1.10^{\mathrm{b}} \pm 0.0$ & $1.1^{\mathrm{b}} \pm 0.0$ & $1.1^{\mathrm{b}} \pm 0.0$ \\
\hline $16: 1$ & $8.3^{\mathrm{a}} \pm 1.0$ & $3.4^{\mathrm{b}} \pm 1.0$ & $2.9^{\mathrm{b}} \pm 1.0$ & $2.5^{\mathrm{b}} \pm 0.7$ & $2.50^{\mathrm{b}} \pm 0.1$ & $2.5^{\mathrm{b}} \pm 0.0$ & $1.7^{\mathrm{b}} \pm 0.1$ \\
\hline $17: 1$ & $0.4^{\mathrm{a}} \pm 0.0$ & $0.5^{\mathrm{a}} \pm 0.0$ & $0.5^{\mathrm{a}} \pm 0.0$ & $0.4^{\mathrm{b}} \pm 0.0$ & $2.4^{\mathrm{b}} \pm 0.1$ & $1.2^{\mathrm{c}} \pm 0.0$ & $1.4^{\mathrm{d}} \pm 0.0$ \\
\hline $18: 1 n-9, n-7$ & $16.3^{\mathrm{a}} \pm 2.0$ & $16.3^{\mathrm{a}} \pm 2.0$ & $17.7^{\mathrm{a}} \pm 2.0$ & $18.2^{\mathrm{a}} \pm 0.8$ & $18.4^{\mathrm{ab}} \pm 1.1$ & $19.3^{\mathrm{ab}} \pm 0.7$ & $19.9^{\mathrm{b}} \pm 1.2$ \\
\hline $20: 1 n-9$ & $7.5^{\mathrm{a}} \pm 0.5$ & $2.1^{\mathrm{b}} \pm 0.5$ & $1.3^{\mathrm{b}} \pm 0.5$ & $1.7^{\mathrm{b}} \pm 0.3$ & $1.2^{\mathrm{b}} \pm 0.0$ & $1.0^{\mathrm{b}} \pm 0.0$ & $0.6^{\mathrm{b}} \pm 0.0$ \\
\hline $22: 1 n-9$ & $5.1^{\mathrm{a}} \pm 1.0$ & $3.9^{\mathrm{ab}} \pm 1.0$ & $3.0^{\mathrm{ab}} \pm 1.0$ & $2.7^{b} \pm 0.3$ & $2.4^{\mathrm{b}} \pm 0.1$ & $1.9^{b} \pm 0.2$ & $1.2^{\mathrm{b}} \pm 0.2$ \\
\hline $24: 1 n-9$ & - & $0.6^{\mathrm{b}} \pm 0.0$ & $1.0^{\mathrm{c}} \pm 0.0$ & $1.1^{\mathrm{d}} \pm 0.0$ & $0.9^{\mathrm{b}} \pm 0.0$ & $1.3^{\mathrm{c}} \pm 0.0$ & $1.0^{\mathrm{d}} \pm 0.0$ \\
\hline $18: 2 n-6$ & $1.5^{\mathrm{a}} \pm 0.2$ & $5.5^{\mathrm{b}} \pm 0.8$ & $8.2^{c} \pm 0.8$ & $9.1^{\mathrm{c}} \pm 0.7$ & $16.1^{\mathrm{b}} \pm 0.9$ & $18.5^{\mathrm{bc}} \pm 1.5$ & $19.4^{\mathrm{c}} \pm 0.9$ \\
\hline $18: 3 n-3$ & $0.6^{\mathrm{a}} \pm 0.2$ & $13.4^{\mathrm{b}} \pm 2.0$ & $14.3^{\mathrm{b}} \pm 2.0$ & $15.5^{\mathrm{b}} \pm 1.0$ & $3.2^{b} \pm 0.3$ & $3.3^{\mathrm{b}} \pm 0.1$ & $3.4^{\mathrm{b}} \pm 0.1$ \\
\hline $20: 2 n-6$ & $0.1^{\mathrm{a}} \pm 0.0$ & $0.2^{\mathrm{a}} \pm 0.0$ & $0.2^{\mathrm{a}} \pm 0.0$ & $0.1^{\mathrm{a}} \pm 0.0$ & $1.2^{\mathrm{b}} \pm 0.0$ & $1.0^{\mathrm{c}} \pm 0.0$ & $0.4^{\mathrm{d}} \pm 0.0$ \\
\hline $20: 3 n-3$ & $2.0^{\mathrm{a}} \pm 0.0$ & $0.4^{\mathrm{b}} \pm 0.0$ & $0.4^{\mathrm{c}} \pm 0.0$ & $0.2^{\mathrm{d}} \pm 0.0$ & $1.4^{\mathrm{b}} \pm 0.0$ & $1.4^{\mathrm{b}} \pm 0.0$ & $1.3^{\mathrm{c}} \pm 0.0$ \\
\hline $20: 4 n-6$ & $2.7^{\mathrm{a}} \pm 0.2$ & $1.3^{\mathrm{b}} \pm 0.2$ & $1.2^{\mathrm{b}} \pm 0.2$ & $1.1^{\mathrm{b}} \pm 0.2$ & $1.2^{\mathrm{b}} \pm 0.2$ & $1.1^{\mathrm{b}} \pm 0.0$ & $0.9^{\mathrm{b}} \pm 0.0$ \\
\hline $20: 5 n-3$ & $5.4^{\mathrm{a}} \pm 1.0$ & $2.3^{\mathrm{b}} \pm 1.0$ & $2.4^{\mathrm{b}} \pm 0.4$ & $2.2^{\mathrm{b}} \pm 0.1$ & $3.9^{\mathrm{b}} \pm 0.2$ & $2.3^{\mathrm{c}} \pm 0.2$ & $1.7^{\mathrm{c}} \pm 0.1$ \\
\hline $22: 2 n-6$ & $1.4^{\mathrm{a}} \pm 0.2$ & $1.1^{\mathrm{ab}} \pm 0.2$ & $0.9^{b} \pm 0.2$ & $0.7^{\mathrm{b}} \pm 0.1$ & $0.9^{b} \pm 0.0$ & $0.6^{b c} \pm 0.0$ & $0.5^{\mathrm{c}} \pm 0.0$ \\
\hline $22: 5 n-3$ & $3.8^{\mathrm{a}} \pm 1.0$ & $2.8^{\mathrm{ab}} \pm 0.2$ & $1.9^{\mathrm{b}} \pm 0.2$ & $1.5^{\mathrm{b}} \pm 0.1$ & $3.5^{\mathrm{a}} \pm 0.4$ & $3.1^{\mathrm{a}} \pm 0.2$ & $2.9^{\mathrm{a}} \pm 0.6$ \\
\hline $22: 6 n-3$ & $11.2^{\mathrm{a}} \pm 1.4$ & $6.0^{\mathrm{b}} \pm 0.8$ & $4.8^{\mathrm{cb}} \pm 0.3$ & $3.2^{\mathrm{c}} \pm 0.2$ & $6.5^{\mathrm{b}} \pm 0.9$ & $5.3^{\mathrm{b}} \pm 0.7$ & $5.0^{\mathrm{b}} \pm 0.8$ \\
\hline SFA & 26.0 & 33.9 & 34.6 & 35.2 & 27.8 & 30.4 & 33.4 \\
\hline MUFA & 39.9 & 28.0 & 27.5 & 27.6 & $29.9^{b}$ & $29.3^{\mathrm{b}}$ & $27.9^{\mathrm{b}}$ \\
\hline PUFA & 28.7 & 33.0 & 34.3 & 33.6 & 37.9 & 36.6 & 35.5 \\
\hline$n$-3Lc PUFA & $22.4^{\mathrm{a}}$ & $11.5^{\mathrm{b}}$ & $9.5^{\mathrm{b}}$ & $7.1^{\mathrm{b}}$ & $15.3^{\mathrm{ab}}$ & $12.1^{\mathrm{b}}$ & $10.9^{\mathrm{b}}$ \\
\hline$n-6$ PUFA & 5.7 & 8.1 & 10.5 & 11.0 & 19.4 & 21.2 & 21.2 \\
\hline
\end{tabular}

SFA saturated FA, MUFA monounsaturated FA, PUFA polyunsaturated FA, LcPUFA long chain polyunsaturated FA.

$80.6 \mathrm{~g}$ TL and $700.5 \mathrm{~g}$ moisture $\mathrm{kg}^{-1} \mathrm{DM}$ ) as compared to CTRL fish $\left(180.4,71.3\right.$ and $\left.700.5 \mathrm{~g} \mathrm{~kg}^{-1}\right)$, followed by LO50 fed fish (Tab. 5).

\subsection{Muscle fatty acid profile}

The effect of feeding incremental levels of either LO or SFO on fatty acids (FA) profile of fish muscles at end of the trial is given in Table 6. It is clear that FA compositions of muscle lipids are closely related to the dietary fatty acid profiles (Tab. 3). As expected, the amount of total saturated fatty acids (SFA) in vegetable oil-fed fish had progressively increased $(P>0.05)$, relative to CTRL, with each increase in vegetable oil level in the diet with the highest concentration in LO70/SFO70 fish among all. Percentage increase ranged between $6.8 \%$ in SFO50-fed, and $30.3 \%$ in LO50-fed fish relative to CTRL fish. Likewise, $n$-6 PUFA recorded elevated values with each rise in vegetable oil dietary percent, compared to CTRL, particularly for SFO fed groups, with a percentage increase of about $42.5 \%$ and $239 \%$ for LO50 and SFO50 groups respectively. On the meantime, monounsaturated FA (MUFA) and $n$-3 LcPUFA had reduced in vegetable oil-fed fish as compared to CTRL fish. A $30.5 \%$ or $24.7 \%$ reduction in MUFA was recorded in LO50 or SFO50 fish respectively, whereas these percentages were $46.8 \%$ or $31 \%$ for total n-3 LcPUFA.

As expected, a substantial increase in linoleic acid (LOA, 18:2 n-6) concentration was detected, in vegetable oil-fed fish relative to CTRL, particularly in SFO-fed fish. In this dietary group linoleic acid (LOA) was the most abundant fatty acid of all PUFAs, whereas in LO-fed fish linolenic acid ( $\alpha$-LNA, $18: 3 n-3)$ was the dominant. Percentage increase of LOA was $965 \%$ in SFO50 fish, and of $\alpha$-LNA was $2296 \%$ in LO50 fish. In comparison to the dietary LOA and $\alpha$-LNA concentrations, the level of these FAs in fish fillet was always lower than the corresponding in their diet for all fish groups (Tab. 3).

As expected, total n-3 LcPUFA decreased gradually with each rise in dietary vegetable oil incorporation level. 


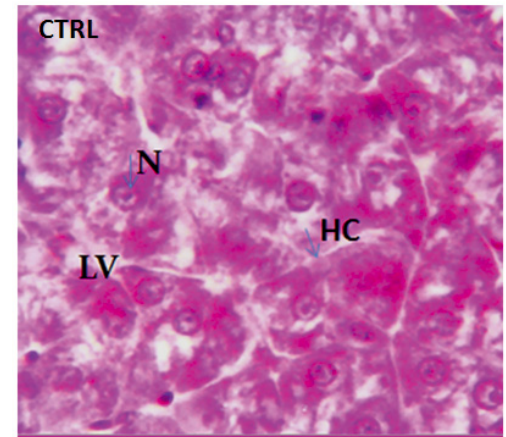

Fig. 1. Cross section in liver of fish fed the CTRL diet, showing normal polygonal hepatocytes $(\mathrm{HC})$ with central round-shape nucleus (N) (H and E, X1000).

While fish fed the CTRL (100\%FO) diet contained the highest concentration of $n$-3 LcPUFA, those fed the LO70/SFO70 diet contained the lowest level. Both EPA and DHA were significantly $(P<0.05)$ decreased in vegetable oil-fed fish relative to the corresponding values of CTRL fish. EPA was reduced by $57.1 \%$ or $28.5 \%$ in LO50 or SFO50 fish respectively, whereas DHA by about $47.1 \%$ or $42.6 \%$ for the same fish groups each in turn. Moreover, DHA levels in muscle lipids (Tab. 6) were always higher than the corresponding in their diet. On the contrary, LOA and $\alpha$-LNA content was lower in fish muscles than in diet, regardless of lipid source or inclusion level (Tab. 3). Unexpectedly, a significantly less concentration of DHA was deposited in the muscle with LO70 diet (3.2\%) than with SFO70 diet $(5.0 \%)$. Percentage reduction of arachidonic acid (ARA, 20:4 $n$-6) was also decreased in SFO50fed fish $(57.4 \%)$ and LO50-fed fish $(53 \%)$ relative to that of CTRL fish.

In general, the best muscle FA profile was that of CTRL followed by SFO50 fish, whereas the worst (lowest $n$-3 LcPUFA and MUFA and highest $n-6$ PUFA and SFA) among all treatments were those of both VO70 groups, particularly the LO70 fish group.

\subsection{Liver histology}

Examination of liver sections from fish fed the experimental diets, for 9 weeks, evidenced a normal histological morphology for only FO-fed fish (CTRL), as well as dietaryinduced changes in hepatic morphology with the graded vegetable oil levels. Hepatocytes (HC) of CTRL fish are hexagonal- shaped with large central nuclei $(\mathrm{N})$ and 1-4 nucleoli within each and contained little lipidic droplets, seen as cytoplasmic vacuoles, corresponding to naturally low lipid content (Fig. 1). No remarkable dietary effect can be observed when comparing the hepatic histological pattern of either LO50- or SFO50-fed fish (Figs. 2a, 2b) with CTRL fish. However, substantial changes in hepatic morphology were found in VO60 fish, as can be observed on the relatively higher hepatocellular vacuolization, being dispersed in the cytoplasm as large droplets (LV) (Figs. 3a, 3b). Fish fed the VO70 diets had more voluminous hepatocytes, due to an accumulation of large lipidic droplets within the cytoplasm, and nucleus displaced to cell periphery (Figs. 4a, 4b). The pyknotic nuclei (PN) and cell organelles shift towards the cell-periphery indicated

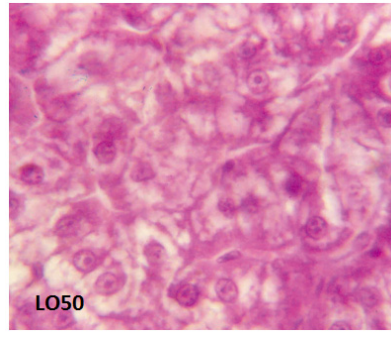

(a)

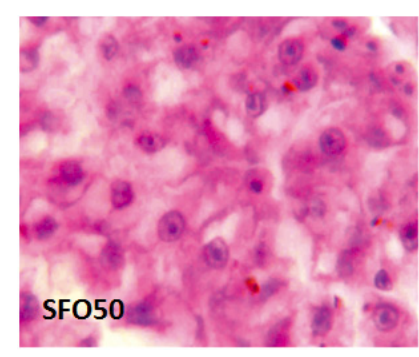

(b)
Fig. 2. Liver of fish fed the LO50 (a) or SFO50 diet (b), showing wellshaped hepatocytes but relatively more cytoplasmic vacuolization or lipidic vacuoles (LV) than in CTRL liver section (H and E, X1000).

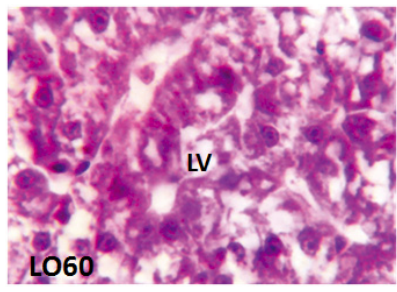

(a)

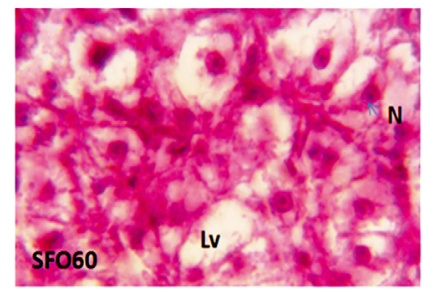

(b)
Fig. 3. Liver of fish fed the LO60 (a) or SFO60 diet (b), showing an increase in lipidic vacuoles (LV) particularly in for the SFO-fed fish (H and E, X1000).

severe lipid infiltration (steatosis or lipoid liver), particularly for SFO70 fish (Fig. 4b). As a general observation, dietaryinduced alterations in hepatic histological scenario were more obvious in SFO- than in LO-fed fish at all added levels (40, 48 and $56 \mathrm{~g} \mathrm{~kg}^{-1}$ diet).

\section{Discussion}

Grain sources of lipids have had an extensive amount of research on their application as an alternative to fish oils in aquaculture feeds. Lipid sources from grain resources have an advantage in that there is often abundant, large-scale production at economically viable costs and they are generally free from potential transmissible pathogens (Bell et al., 2005). However, despite significant levels of $n-3$ and $n-6$ fatty acids being present in these resources, their key limitation is that none of them possess any of the highly bioactive LcPUFA, such as DHA, EPA or ARA (reviewed by Turchini et al., 2009; Glencross, 2009). Numerous research has been carried out on the substitution of fish oil by vegetable oils within compounded fish feeds, using fishmeal-based diets, high-energy diets or plant-protein rich diets and results were always promising. Linseed oil (LO) is among the most common vegetable oils examined, singly or in combination, in researches for fish feed production including gilthead seabream (reviewed by Nasopoulou and Zebatakis, 2012), whereas sunflower oil (SFO) was not previously evaluated for the species. However, reports on using sunflower oil in feeds for Atlantic salmon (Salmo salar) (Torestensen et al., 2000; Bransden et al., 2003; Menoyo et al., 2007) and European seabass (Dicentrarchus labrax) (Yieldiz and Sener, 2004) are promising. 


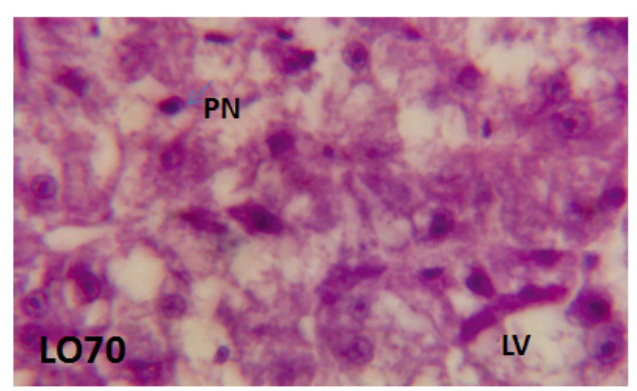

(a)

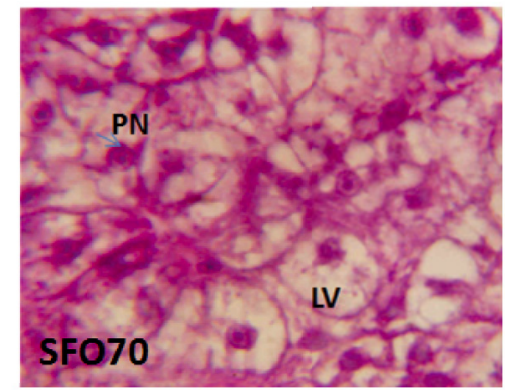

(b)

Fig. 4. Liver of fish fed the LO70 (a) or SFO70 diet (b), showing swelling hepatocytes (HC) with severe fatty infiltration caused larger and intense cytoplasmic vacuolization (LV) and pyknotic nulei (PN) shifted towards cell-periphery $(\mathrm{H}$ and $\mathrm{E}, \times 1000)$.

In the present study, first report, incremental levels of either SFO or LO (40-56 $\mathrm{g} \mathrm{kg}^{-1}$ diet) were individually evaluated as partial substitutes to fish oil in gilthead seabream fingerling feeds. Despite the different FO/VO combination of tested diets, all were almost equally consumed by fish, but with a lesser degree for LO-added diets. In terms of growth rate, fish fed the $40 \mathrm{~g}$ FO plus $40 \mathrm{~g} \mathrm{SFO}$ or $\mathrm{LO} \mathrm{kg}^{-1}$ diet has performed the best and retained the highest nutrient composition in muscles among all, therefore preferably suggested. However, in terms of cost, a combination of $32 \mathrm{~g}$ FO plus $48 \mathrm{~g} \mathrm{LO}$ or SFO kg-1 diet can be also used with the least consequences on growth, feed utilization and nutrient retention efficiency. This notion is higher than the maximum level of FO-substitution by soybean oil $(47 \%)$ in gilthead seabream juveniles diet (Kalogeropoulos et al., 1992) and closely resemble to that for cottonseed oil (Wassef et al., 2014) or a blend of soybeen, linseed and cottonseed oils (Wassef et al., 2007, 2009).

Under our experimental conditions, the growth rate obtained is considered satisfactory for this size of fish, and are comparable or higher than those previously reported for the species (Beneditto-Palos et al., 2008; Caballero et al., 2004; El-Kerdawy and Salama, 1997; Izquierdo et al., 2003, 2005). It is well documented that, partial replacement of fish oil by vegetable would only be possible if essential fatty acids (EFAs) (primarily $n-3$ LcPUFA) in the diet were present in sufficient amounts to cover fish's need. For gilthead seabream, the minimum requirements for EPA and DHA were estimated to be around $0.9 \%$ of the diet (Kalogeropoulos et al., 1992; Izquierdo, 2005). It is clear, from the fatty acid profile of the experimental diets of this study (Tab. 3) that EPA and DHA amounts are enough to meet requirements for this species. Prior studies have examined the use of LO in gilthead seabream diets, as a partial substitute of fish oil, had reported different inclusion levels associated with the use of both dietary protein and lipid sources. In fishmeal-based diets, $50 \%$ and $60 \%$ FO-substitution by LO either singly (46\% CP and $25 \% \mathrm{~L})$ or in a blend $(46 \% \mathrm{CP}$ and $18 \% \mathrm{~L})$ can produce similar results to diets containing $100 \%$ fish oil during the grow-out phase of this species (El-Kerdawy and Salama, 1997; Izquierdo et al., 2003; Wassef et al., 2009). In addition, within a plant protein-rich diets higher ratio of added-fish oil $(66 \%)$ was substituted by a mixture of vegetable oils, contained LO, without compromising growth performance or feed efficiency of fish (Benedito-Palos et al., 2008). On the other hand, more higher levels of dietary fish oil substitution up to
$70 \%$ (equivalent to $56 \mathrm{~g} \mathrm{LO} \mathrm{kg}^{-1}$ diet) (Present study) or to $80 \%$ even in high energy diets (25\% lipids) (Montero et al., 2003 ) or to $100 \%$ in plant protein-rich diets (Benedito-Palos et al., 2008) had all resulted in a remarkably reduced growth rate, feed efficiency and inferior muscle major nutrients content than those fed only-fish oil diet. The reduced fish performance of VO70 fed fish, in the present study, may suggest that $n-3$ LcPUFA in these diets had dropped to less than $10 \mathrm{~g} \mathrm{~kg}^{-1}$ and may have induced an EFA limitation. This result may not necessarily be a reflection on SFO or LO impact, but perhaps more on the level of $n$-3 LcPUFA that were available in the diet with the highest inclusion level of each. Ibeas et al. (1996) estimated the optimal dietary level of $n-3$ LcPUFA in juvenile gilthead seabream to be $10 \mathrm{~g} \mathrm{~kg}^{-1}$ and further increases worsening the performance of the fish. Similarly, Kalogeropoulos et al. (1992) estimated the required $n-3$ LcPUFA for this species in diets, with approximately $80-100 \mathrm{~g} \mathrm{~kg}^{-1}$ of lipid, to be approximately $19-23 \%$ of total fatty acids. These fatty acids have a sparing effect of protein in fish, and fish need these fatty acids for normal growth and a better feed conversion ratio (Watanabe, 1982). Ibeas et al. (1997) also found that growth was influenced by the ratio between EPA and DHA, but that optimal growth occurred at a ratio of 2:1 in juveniles or larger fish.

The chemical composition of fish flesh is greatly influenced by fish feed. Lipids in the feeds accumulate in the muscles, viscera and liver after energy metabolism in fish (reviewed by Turchini et al., 2009). The present study showed that viscero-somatic index (VSI) calculated from gilthead seabream fed the SFO70 or LO70 diets were significantly higher than that of fish fed only-FO diet, whereas hepatosomatic index (HSI) was almost similar among treatments indicating higher selective certain lipid deposition in the viscera, than in the liver, of vegetable oil-fed fish. This finding in contrast with that reported for European seabass fed SFO among other vegetable oils, and liver was the preferential fat depot than viscera (Yieldiz and Sener, 2004). Differences in the reported effects on liver, viscera or muscles lipid stores are mainly species specific, but can also result from differences in fish size and the duration of the feeding experiments.

In the present study, as for most fatty acids studies, fatty acids accumulating in the muscle of fish largely reflected that of their respective diets. Distinct differences were noted between the all fish oil- and vegetable oil-added dietary groups particularly in the proportions of LOA and $\alpha$-LNA and DHA. 
The use of LO60/SFO60 diets has altered the nutritional quality of gilthead seabream, reducing the levels of $n-3$ LcPUFA, primarily EPA and DHA, and increasing levels of LOA and $\alpha$-LNA (Tab. 6). These results are in accordance with those earlier reported for European seabass, that LAO was found in higher levels in SFO- or soybean oil (SBO)-fed fish than in the other groups (Yieldiz and Sener, 2004). However, virtually all studies on this species reported changes in the tissue fatty acid composition, most notably a rise in $n-6$ series fatty acids (reviewed by Glencross, 2009; Nasopoulou and Zebatakis, 2012). This indicated that gilthead seabream have limited capabilities to convert LOA and $\alpha$-LNA into EPA, DHA and ARA. These findings are in accordance with those reported by Seiliez et al. (2003) for marine fish in general. However, Izquierdo et al. (2003) suggested possible FA chain elongation and desaturation of $\alpha$-LNA in this species which might have low levels of $\Delta^{6}$ and $\Delta^{5}$ desaturase activity.

The relatively lower concentrations of LOA and $\alpha$-LNA in fish muscles fed either LO60/SFO60 diet of the present study, compared to their levels within the corresponding diet suggest that gilthead seabream had utilized these fatty acids for oxidation. Furthermore, the increase in the levels of DHA from fish fed diets with low dietary levels of this fatty acid has led to speculate that DHA was selectively retained in fish muscles compared to other fatty acids. Similar observations were also reported for bigger sizes of the species $(\sim 130 \mathrm{~g} / \mathrm{fish})$ fed a vegetable oils blend for 20 weeks (Wassef et al., 2009, 2012) and for European seabass (Dicentrarchus labrax) as well (Mourente et al., 2005). In the present study, the relatively less DHA deposition in the muscle with LO70 diet than with SFO70 diet (Tab. 6), despite their almost equal DHA content, may be due to the different pathway of each diet. Most studies examining the use of alternative oils to fish oil, at certain inclusion level, have shown no reduction in growth, but have shown significant alterations in the fatty acid composition of fish tissues. This fatty acid composition shift has prompted some researchers to suggest the need for 'finisher' diets as a means of restoring the muscle FA profile back to the profile present when the fish were re-fed diets using only fish oil. Therefore, these changes in muscle fatty acid composition after fish being fed a LO or SFO diets can be restored after re-feeding gilthead seabream a fish oil-finishing diet at end of the production cycle, for 90 days (Izquierdo et al., 2005) or 120 days (Fountoulaki et al., 2009).

In addition, both LO and SFO was further evaluated in feeds for other fish species. Bell et al. (1993) evaluated SFO and LO versus FO for Atlantic salmon (Salmo salar) as the added lipid component in a study that examined the phospholipid and eicosanoid chemistry and cardiac health of fish. Fish fed the SFO diet had marked decreases in ARA, EPA and DHA, while fish fed the LO diet had an increased ARA content. These results contradict partially with present study's which revealed a reduction in ARA percent when fish fed either LO- or SFO-diets compared to all-FO diet (Tab. 6). In another study, Bransden et al. (2003) suggested that only $40 \%$ of dietary FO of Atlantic salmon could be replaced by SFO before a significant reduction in the flesh concentration of DHA resulted. Menoyo et al. (2007) examined the influence of replacing either FO or LO with SFO in four levels of replacement $(0 \%, 25 \%, 50 \%$ and $75 \%)$ in diets fed to Atlantic salmon, and found no differences in growth among treatments, however, lipid digestibility was affected by the level of saturated fatty acids (SFA) in the diet. These earlier studies provide support for the use of either SFO or LO as a complementary lipid resource in Atlantic salmon diets, and the present study additionally in gilthead seabream diets.

Incorporation of vegetable oils in marine fish diets may lead to imbalance in the essential or non-essential FAs, affecting liver tissue integrity (Caballero et al., 2004). In the meantime, feeding gilthead seabream over $40 \mathrm{~g} \mathrm{VO} \mathrm{kg}^{-1}$ diet (SFO60 or LO60 diets), in the present study, has further resulted in dense accumulation of lipid droplets within the hepatocytes, more obvious in SFO-fed fish (Figs. 2 and 3), different from normal histological structure of CTRL fish liver (Fig. 1). This phenomenon might be in relation to decreased levels of dietary n-3 LcPUFA. Lipoid livers, due to severe fatinfiltration, were also shown after high levels of LO or SFO intake (LO/SFO70 diets) especially for SFO-fed fish, indicating that dietary lipid exceeded the capacity of the hepatic cells to oxidize fatty acids, or due o protein synthesis impairment (Watanabe, 1982) which resulted in large synthesis and deposition of triglycerides (TAGs) in vacuoles, leading to that morphological pattern known as steatosis or lipoid liver. Results of the present study are in broad agreement with earlier findings in other fish species such as European seabass (Mourente et al., 2005). They reported that accumulation of lipid droplets in the livers of fish fed LO and rapeseed oil (RO) diets may be due to the preference for specific FAs as energy sources resulting in storage of surplus FAs (e.g. 18:3n-3 and 18:1 n-9) in lipid droplets. Likewise, this phenomenon is not pathogenic but reversal when fish re-fed a FO-finishing diet (Caballero et al., 2004) and eventually highlighted the importance of $n$ - 3 LcPUFA in gilthead seabream feeds.

In conclusion, results of feeding gilthead seabream fingerling diets contained either LO or SFO, in levels ranging between 40 and $56 \mathrm{~g} \mathrm{~kg}^{-1}$ diet, indicated that both vegetable oils have shown a sound ability to replace added FO, up to $48 \mathrm{~g} \mathrm{~kg}^{-1}$ diet utmost for further cost reduction, with limited effects on growth and feed utilization, but with notable effects on the fatty acid composition of the fish and liver integrity. A preference for SFO- over LO-diets in terms of growth performance and cost was evidenced and vice versa for liver structure.

\section{References}

Alexis MN. 1997. Fish meal and fish oil replacers in Mediterranean marine fish diets. In: Tacon A, Basurco B eds. Proc. Workshop "Feeding Tomorrow's Fish". Mazarron, Spain, 1996. Cahiers Options Méditerranéenes 22: 183-204.

AOAC. 1995. Association of Official Agricultural Chemists. Official Methods of Analysis. 16th ed. Arlington, VA, USA.

Bell JG, Dick JR, Sargent JR. 1993. Effects of diets rich in linoleic or alpha-linolenic acid on phospholipid fatty acid composition and eicosanoid production of Atlantic salmon (Salmo salar). Lipids 26: 445-450.

Bell JG, McGhee F, Dick JR, Tocher DR. 2005. Dioxin and dioxinlike polychlorinated (PCBs) in Scottish farmed salmon (Salmo salar): effects of replacement of dietary marine fish oil with vegetable oils. Aquaculture 243: 305-314. 
Benedito-Palos L, Navarro JC, Sitjá-Bobadilla A, Bell JG, Kaushik SJ, Pérez-Sánchez J. 2008. High levels of vegetable oils in plant protein-rich diets fed to gilthead seabream (Sparus aurata L.): growth performance, muscle fatty acid profiles and histological alterations of target tissues. Br. J. Nutr. 100: 992-1003.

Benedito-Palos L, Navarro JC, Bermejo-Nogales A, Saera-Vila A. 2009. The time course of fish oil wash-out follows a simple dilution model in gilthead seabream (Sparus aurata L.) fed graded levels of vegetable oils. Aquacul. 288: 98-105.

Benedito-Palos L, Bermejo-Nogales A, Karampatos AI, et al. 2011. Modeling the predictable effects of dietary lipid sources on the fillet fatty acid composition on one-year-old gilthead seabream (Sparus aurata L.). Food Chem. 124: 538-544.

Bransden MP, Carter CG, Nichols PD. 2003. Replacement of fish oil with sunflower oil in feeds for Atlantic salmon (Salmo salar L.): effect on growth performance, tissue fatty acid composition and disease resistance. Comp. Biochem. Physiol. B 135: 611-625.

Caballero MJ, Izquierdo MS, Kjørsvik E, Fernandez AJ, Rosenlund G. 2004. Histolo-gical alterations in the liver of seabream, Sparus aurata L., caused by short- or long-term feeding with vegetable oils. Recovery of normal morphology afterfeeding fish oil as the sole lipid source. J. Fish Dis. 27: 531-541.

Christie WW. Isolation, separation, identification and structural analysis of lipids. In: Lipid Analysis. 2nd ed. Oxford, England: Pergamon Press, 1982, p. 207.

Diaz-Lopéz M, Perez MJ, Acosta NG, et al. 2009. Effect of dietary substitution of fish oil by Echium oil on growth, plasma parameters and body lipid composition in gilthead seabream (Sparus aurata L.). Aquacul. Nutr. 15: 500-512.

Glencross BD. 2009. Exploring the nutritional demand for essential fatty acids by aquaculture species. Reviews in Aquaculture 1: 71-124.

El-Kerdawy A, Salama M. 1997. Effect of dietary lipid sources on the growth and fatty acid composition of gilthead seabream (Sparus aurata). In: Tacon A, Basurco B Eds. Proc. Workshop "Feeding Tomorrow's Fish". Mazarron, Spain, June 24-26 1996. Cahier Options Méditerranéennes 22: 235-242.

Folch JM, Lee SM, Sloane-Stanley GH. 1957. A simple method for the isolation and purification of total lipids from the animal tissues. J. Biol. Chem. 226: 497-509.

Fountoulaki E, Vasilaki A, Hurtado R, et al. 2009. Fish oil substitution by vegetable oils in commercial diets for gilthead seabream (Sparus aurata L.): effects on growth performance, flesh quality and fillet fatty acid profile. Recovery of fatty acid profiles by a fish oil finishing diet under fluctuating water temperatures. Aquacul. 289: 317-326.

Goddar S. (ed.) 1996. Feed Management in Intensive Aquaculture. p. 45. Chapman and Hall Publisher, p. 194.

Ibeas C, Cejas J, Gomez T, Jerez S, Lorenzo A. 1996. Influence of $n-3$ highly unsaturated fatty acid levels on juvenile gilthead seabream (Sparus aurata) growth and tissue fatty acid composition. Aquacul. 142: 221-235.

Ibeas C, Cejas JR, Fores R, Badia P, Gomez T, Lorenzo A. 1997. Influence of the eicosapentaenoic to docosahexaenoic acid ratio (EPA/DHA) of dietary lipids on growth and fatty acid composition of gilthead seabream (Sparus aurata) juveniles. Aquacul. 150: 91-102.
Izquierdo MS. 2005. Essential fatty acid requirements in Mediterranean fish species. Cahier Options Méditerranéennes. Special Issue 'Mediterranean Fish Nutrition' 63: 91-102.

Izquierdo MS, Obach A, Arantzamendi L, Montero D, Robaina L, Rosenlund G. 2003. Dietary lipid sources for seabream and seabass: growth performance, tissue composition and flesh quality. Aquacul. Nutr. 9: 397-407.

Izquierdo MS, Montero D, Robaina L, Cabalerro MJ, Rosenlund G, Giné R. 2005. Alterations in fillet fatty acid profile and flesh quality in gilthead seabream (Sparus aurata) fed vegetable oils for a long term period. Recovery of fatty acid profiles by fish oil feeding. Aquacul. 250: 431-444.

Kalogeropoulos N, Alexis MN, Henderson RJ. 1992. Effects of dietary soybean and cod-liver oil levels on growth and body composition of gilthead seabream (Sparus aurata). Aquacul. 104: 293-308.

Kaushik SJ. 1997. Nutritional and the improvement of seabass and seabream production in the Mediterranean region. Recent Development in the Nutrition and Feeding of Marine Finfish of Interest to Mediterranean. ALIIA Trades Show, September 27, Thessaloniki, Greece.

Menoyo D, Izquierdo MS, Robaina L, Ginés R, López-Bote CJ, Bautista JM. 2004. Adaptation of lipid metabolism, tissue composition and flesh quality in gilthead seabream (Sparus aurata) to fish oil replacement by linseed and soybean oils. British J. Nutr. 92: 41-52.

Menoyo D, Lopez-Bote CJ, Diez A, Obach A, Bautista JM. 2007. Impact of $n-3$ fatty acid chain length and $n-3 / n-6$ ratio in Atlantic salmon (Salmo salar) diets. Aquacul. 267: 248-259.

Montero D, Kalinowski T, Obach A, et al. 2003. Vegetable lipid sources for gilthead seabream (Sparus aurata): effects on fish health. Aquacul. 225: 353-370.

Mourente G, Good JE, Bell JG. 2005. Partial substitution of fish oil with rapeseed, linseed and olive oils in diets for European seabass (Dicentrarchus labrax L.): effects on flesh fatty acid composition, plasma prostaglandin E2 and F2 $\alpha$, immune function and effectiveness of a fish oil finishing diet. Aquacul. Nutr. 11: 25-40.

Nasopoulou C, Zebatakis I. 2012. Benefits of fish oil replacement by plant originated oils in compounded fish feeds. A review. LWTFood Sci. Technol. 47: 217-224.

Nykter M, Kymäläinen H-R, Gates F, Sjöberg A-M. 2006. Review. Quality characteristics of edible linseed oil. Agr. Food Sci. 15: 402-4013.

Seiliez L, Panserat S, Corraze G, Kaushik SJ, Bergot P. 2003. Cloning and nutritional regulation of a $\Delta 6$-desaturate-like enzyme in the marine teleost gilthead seabream (Sparus aurata). Comp. Biochem. Physiol. B 135: 449-460.

Shearer KP. 1994. Factors affecting the proximate composition of cultured fishes with emphasis on salmonids. Aquacul. 119: 63-88.

Tocher DR, Harvie DA. 1988. Fatty acid composition of the major phosphoglycerides from fish neural tissues: $(n-3)$ and $(n-6)$ polyunsaturated fatty acids in rainbow trout (Salmo gairdneri L.) and cod (Gadus morhua) brains and retinas. Fish Physiol. Biochem. 5: 229-239.

Torestensen BE, Øyvind L, Frøyland I. 2000. Lipid metabolism and tissue composition in Atlantic salmon (Salmo salar L.)- effects of capelin oil, palm oil and oleic acid-enriched sunflower oil as dietary lipid sources. Lipids 35: 653-664.

Turchini GM, Torstensen BE, Ng WK. 2009. A Review: Fish oil replacement in finfish nutrition. Reviews in Aquaculture 1: 10-57. 
Wassef EA, Wahby OM, Sakr EM. 2007. Effect of dietary vegetable oils on health and liver histology of gilthead seabream (Sparus aurata) growers. Aquac. Research 38: 1365-2109.

Wassef EA, Saleh NE, El-Abd El-Hady HA. 2009. Vegetable oil blend as alternative lipid resource in diets for gilthead seabream, Sparus aurata. Aquacul. Int. 17: 421-435.

Wassef EA, Shalaby SH, Wahby OM. 2012. Effects of dietary vegetable oils on liver and gonad fatty acid metabolism and gonad maturation in gilthead seabream (Sparus aurata) males and females. Aquacul. Int. 20: 255-281.
Wassef EA, Shalaby SH, Saleh NE. 2014. Cottonseed oil as a complementary lipid source in diets for gilthead seabream (Sparus aurata) juveniles. Aquac. Research, Doi : 10.1111/are. 12405.

Watanabe T. 1982. Lipid nutrition in fish. Comp. Biochem. Physiol. B 73: $3-15$.

Yildiz M, Sener E. 2004. The effects of dietary oils of vegetable origin on the performance, body composition and fatty acid profiles of seabass (Dicentrarchus labrax L. 1758) juveniles. Turk. J. Vet. Anim. Sci. 3: 111-116.

Cite this article as: Elham A. Wassef, Shaymaa H. Shalaby, Norhan E. Saleh. Comparative evaluation of sunflower oil and linseed oil as dietary ingredient for gilthead seabream (Sparus aurata) fingerlings. OCL 2015, 22 (2) A201. 